PharmacoEconomics \& Outcomes News 862, p36 - 19 Sep 2020

\title{
Scottish Medicines Consortium September 2020 decisions
}

In September 2020 decisions, the Scottish Medicines Consortium (SMC) has accepted additional new medicines for use within $\mathrm{NHS}^{*}$ Scotland, and three treatments for interim use.

Cannabidiol [Epidyolex] was accepted for the treatment of Lennox-Gastaut syndrome and Dravet syndrome, after consideration through SMC's Patient and Clinician Engagement (PACE) process. Cannabidiol in combination with clobazam has the potential reduce the frequency of seizures in patients with these epilepsy disorders and improve quality of life.

Esketamine [Spravato] was accepted for use in combination with other antidepressants in adults with treatmentresistant major depression after failure of two or more previous treatments. It is administered as a nasal spray under supervision by a healthcare professional.

Pembrolizumab [Keytruda] was accepted through the PACE process for the treatment of metastatic or inoperable head and neck squamous cell carcinoma. It offers the potential for improvement in disease control, quality of life and survival duration. Pembrolizumab was also accepted for the treatment of advanced renal cell carcinoma, in combination with axitinib, following consideration through the PACE process.

Gilteritinb [Xospata] was accepted through the PACE process for the treatment of relapsed or refractory acute myeloid leukaemia in patients with FLT3 mutation. This oral therapy has been shown to increase overall survival, and it has the potential to increase the chance of suitability for stem cell transplantation.

Fluocinolone acetonide intravitreal implant [Iluvien] was accepted for the prevention of relapse inpatients with recurrent non-infectious uveitis. The implant is injected into the eye and reduces the chance of uveitis recurrence over a 3-year period.

The SMC has also accepted the following medicines through an expedited approach to minimise delay in patient access during the COVID-19 pandemic: pertuzumab [Perjeta] for adjuvant treatment of early breast cancer; caplacizumab [Cablivi] for acquired thrombotic thrombocytopenic purpura; zirconium silicate [sodium zirconium cyclosilicate; Lokelma] for the treatment of hyperkalaemia; and brolucizumab [Beovu] for the treatment of wet agerelated macular degeneration.

In addition, the following medicines have been accepted for interim use subject to ongoing evaluation and reassessment by the SMC: andexanet-alfa [Ondexxya] for life-threatening or uncontrolled bleeding in adults receiving certain anticoagulants; polatuzumab [Polivy] for lymphoma in patients unsuitable for a stem cell transplant; and Holoclar stem cell treatment for the replacement of damaged eye cells after chemical or physical burn injuries.

* National Health Service

Scottish Medicines Consortium. September 2020 decisions news release Internet Document : 7 Sep 2020. Available from: URL: https://www.scottishmedicines.org.uk/aboutus/latest-updates/september-2020-decisions-news-release/ 